Provided for non-commercial research and education use. Not for reproduction, distribution or commercial use.

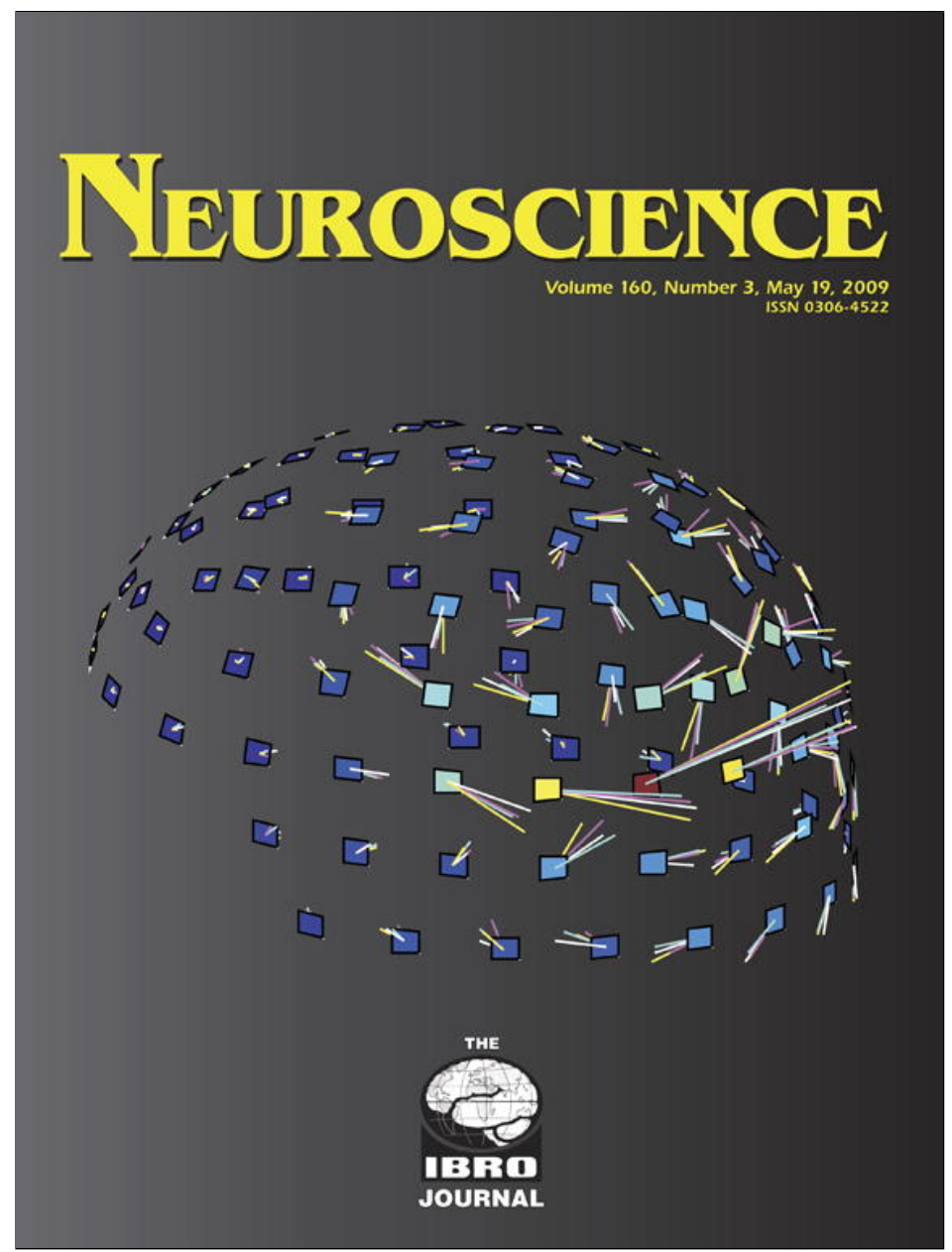

This article appeared in a journal published by Elsevier. The attached copy is furnished to the author for internal non-commercial research and education use, including for instruction at the authors institution and sharing with colleagues.

Other uses, including reproduction and distribution, or selling or licensing copies, or posting to personal, institutional or third party websites are prohibited.

In most cases authors are permitted to post their version of the article (e.g. in Word or Tex form) to their personal website or institutional repository. Authors requiring further information regarding Elsevier's archiving and manuscript policies are encouraged to visit:

http://www.elsevier.com/copyright 


\section{PRECISE TIMING IN FLY MOTION VISION IS MEDIATED BY FAST COMPONENTS OF COMBINED GRADED AND SPIKE SIGNALS}

\section{U. BECKERS, ${ }^{*} M$. EGELHAAF AND R. KURTZ}

Bielefeld University, Department of Neurobiology, Postfach 1001 31, 33501 Bielefeld, Germany

\begin{abstract}
Firing of an individual neuron is determined by the activity of its presynaptic input ensemble. In this study we analyzed how presynaptic signals with different dynamics interact to control postsynaptic activity. In the blowfly's visual system we simultaneously recorded in vivo from an identified motion-sensitive neuron and from elements of the presynaptic ensemble. The presynaptic cells themselves are mutually electrically coupled and convey both graded and spike signals to their common postsynaptic target. We elicited spikes in the postsynaptic neuron by voltage-clamping one of the presynaptic neurons to various holding potentials and then analyzed the time course of the holding current. Current transients in the clamped presynaptic cell were found to coincide with postsynaptic spikes. The current transients were highly variable in amplitude and occasionally absent during postsynaptic spiking. These characteristics indicate that the current transients in the voltage-clamped neuron result from spikes in electrically coupled co-members of the presynaptic ensemble. Our results suggest that electrical coupling among presynaptic neurons mediates synchronization of spikes within the cell ensemble. Moreover, our findings demonstrate that the graded response component of the presynaptic cells effectively controls the postsynaptic firing rate on a coarse scale while the precise timing of the postsynaptic spikes is a consequence of spikes superimposed on the graded signals of the presynaptic neurons. () 2009 IBRO. Published by Elsevier Ltd. All rights reserved.
\end{abstract}

Key words: visual motion pathway, synaptic transmission, synchronization, electrophysiology, voltage clamp, dual recording.

The reliability and performance of neuronal systems is crucially affected by signal transmission and processing at synapses. Relevant issues are the way synapses operate as temporal filters and in how far precise timing information is maintained during synaptic signal transfer. Mostly in invertebrates, for example in leech segmental ganglia, crustacean stomatogastric ganglia or in insect visual pathways, but also in vertebrate nervous systems, neurons are known that convey information by signals that combine graded potential shifts with spikes of variable amplitude, termed in the following "mixed potential signals" (e.g. Hengstenberg, 1977; Graubard et al., 1980; Simmons, 1982; Arbas and Calabrese, 1987; Juusola et al., 1995; Awatramani et al., 2005; de Polavieja et al., 2005; Alle and

*Corresponding author. Tel: +49-521-106-5746.

E-mail address: ubeckers1@uni-bielefeld.de (U. Beckers).

Abbreviation: VS, vertical system.
Geiger, 2006; Shu et al., 2006; Juusola et al., 2007). It is tantalizing to suggest that coarse information (analogous to spike rate) may be carried by the graded component while temporally precise information is present in the timing of spikes of these mixed potential neurons.

In general, comparison of pure spike coding and pure graded coding in the fly visual system has revealed that graded coding can carry more information than purely spike-mediated coding (De Ruyter van Steveninck and Laughlin, 1996; Haag and Borst, 1997; Juusola and French, 1997; Warzecha and Egelhaaf, 2001). A combination of graded and spike-like signals in the axonal membrane potential has been shown to maximize information transmission (Haag and Borst, 1998; de Polavieja et al., 2005; Juusola et al., 2007).

However, in such a mixed potential coding scheme synaptic transmission of the neuronal information is much more demanding than signal transmission in neurons that convey either only graded potentials or spikes. Reliable synaptic transfer of spikes with millisecond precision is often facilitated by nonlinear mechanisms, for example high voltage thresholds of presynaptic $\mathrm{Ca}^{2+}$ channels (Borst and Sakmann, 1998) and a supralinear dependency of transmitter release on presynaptic $\mathrm{Ca}^{2+}$ concentration (Dodge and Rahaminoff, 1967; Smith et al., 1985; Bollmann et al., 2000). These kinds of expansive nonlinearities attenuate presynaptic signals with small amplitude and would therefore be in conflict with the constraint of graded synapses to faithfully transmit also small-amplitude signals (Witkovsky et al., 1997; Ivanov and Calabrese, 2000; Thoreson et al., 2004). So far, it has not been analyzed systematically to what extent timing precision of postsynaptic spikes is influenced at mixed potential synapses by the presence of a concomitant strong graded input.

The synapses investigated in the present study connect part of the blowfly's vertical system cells (VS cells) with the V1 cell (Kurtz et al., 2001). The VS cell ensemble consists of 10 cells, which each integrate outputs of many local retinotopically organized motion-sensitive elements (Hengstenberg, 1982; Krapp et al., 1998). The integrated postsynaptic signals are conveyed to the presynaptic terminals as graded potentials, superimposed by spikes of variable amplitude (Hengstenberg, 1977). VS cells possess large and unique, but overlapping, receptive fields (Hengstenberg et al., 1982; Krapp et al., 1998) and are weakly serially coupled by electrical synapses (Haag and Borst, 2004, Fig. 1). V1 gets strong input from three VS cells with fronto-lateral receptive fields (VS1-VS3) and additional weak and potentially indirect input from further VS cells with more lateral receptive fields (Warzecha et al., 


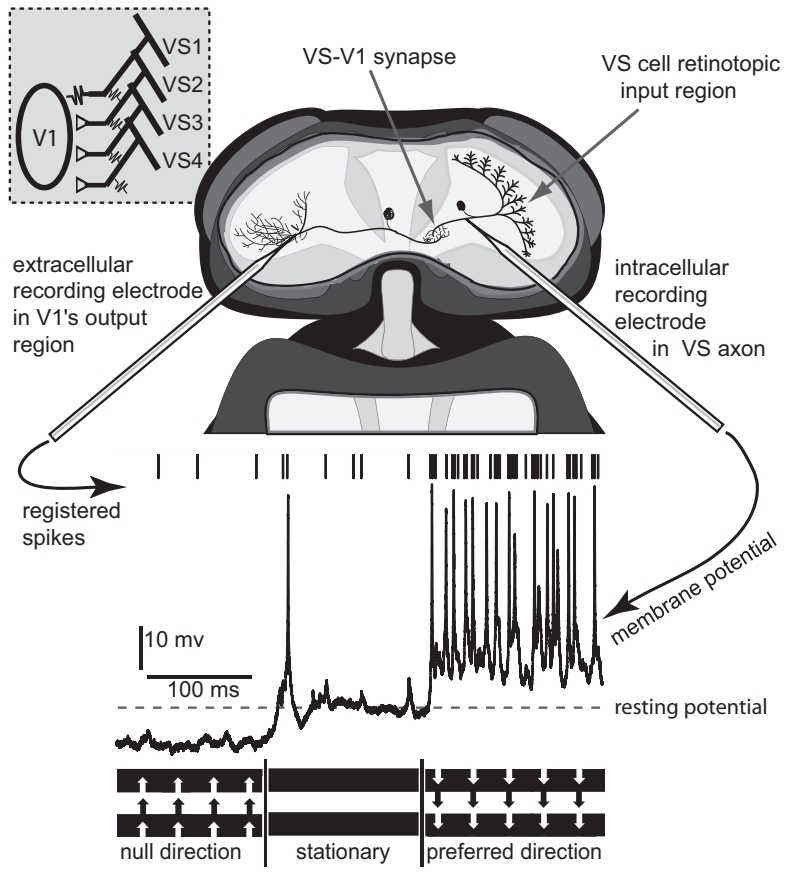

Fig. 1. Schematic of synaptic coupling between VS cells and the V1 cell in the fly visual-motion pathway (top) and example traces of a dual recording of one VS cell and V1 during visual motion stimulation (bottom). The VS cells are located in the fly's lobula plate (scheme shows opened head capsule from a caudal perspective) and pool the input of many retinotopically organized small motion-sensitive elements at their large dendrites. The signal is conducted to the axon terminal of VS where it is transferred to V1, which branches in the contralateral brain hemisphere. V1 is thought to receive direct input from three or 4 out of 10 VS neurons (Kurtz et al., 2001; Warzecha et al., 2003; Kalb et al., 2006) that are electrically coupled with their respective neighbors (Haag and Borst, 2004). The inset shows the most probable synaptic wiring scheme according to Kalb et al. (2006). For simplicity only one VS cell is shown in the scheme. VS cells were recorded intracellularly in their axon near the output region to guarantee a close correspondence of the synaptic potential by the voltageclamp command potential. V1 was recorded extracellularly at its contralateral output region. During constant-velocity motion of a grating in the null direction (upward) VS cells respond with graded hyperpolarizations. Upon cessation of stimulus motion rebound spikes may be elicited and the potential quickly recovers to resting potential. Constant-velocity motion stimulation in the preferred direction (downward) elicits graded depolarizations on which spikes of variable amplitude are superimposed in the VS neuron. The purely spiking cell V1 reduces firing rate below resting activity during null-direction motion and increases its spike rate in response to preferred-direction motion (vertical lines in the upper trace denote V1 spikes). Note the coincidence of V1 spikes with fast depolarizing transients in VS.

2003; Kalb et al., 2006). In contrast to VS, the V1 cell transmits information to its output region exclusively by full-blown action potentials (Hausen, 1976).

Synaptic transmission between VS and V1 has recently been shown to be almost linear for presynaptic voltage fluctuations over a broad range of signal amplitudes and dynamics (Warzecha et al., 2003; Beckers et al., 2007). This frequency transmission range is broader than the frequency range over which velocity fluctuations of a stimulus pattern are linearly encoded (Haag and Borst, 1997; Warzecha et al., 1998). The latter is limited due to the low-pass filters and nonlinear computations that are thought to form necessary constituents of the cellular processes underlying visual motion detection (Borst and Egelhaaf, 1989; Egelhaaf and Borst, 1989). Nonetheless, in addition to transmission of graded signals, synapses between VS and V1 are able to transmit information carried by presynaptic spikes. This has been concluded from the observation of a close temporal correlation between presynaptic and postsynaptic spikes during visual stimulation with white noise velocity modulations (Warzecha et al., 2003).

The nature of synapses between VS cells and the V1 cell is still unclear. Though anatomical specializations as are typical of chemical synapses were visible at output arborizations of VS cells (Hausen et al., 1980), it is not yet known whether individual synapses of the different VS cells to V1 are exclusively either chemical or electrical or even mixed chemical and electrical synapses. Mixed synapses comprising chemical and electrical contact sites in close vicinity have been demonstrated to be present in the connections that a subgroup of the VS cells (VS4-9) forms with the descending neurons of the ocellar and vertical system (DNOVS) neurons (Strausfeld and Bassemir, 1985). In a previous study the impact of laser ablation of individual VS cells on V1's spiking activity complied best with electrical synapses between V1 and VS1 but chemical synapses between VS2/3 and V1 (Kalb et al., 2006).

In the present study we use simultaneous dual recordings and voltage-clamp techniques to control the potential of one presynaptic VS cell while registering the spike response of the postsynaptic V1 cell. Since VS cells are electrically coupled with one another (Haag and Borst, 2004) the holding current applied to clamp the voltage of one of the VS cells reveals the presence of spikes in neighboring VS cells. We exploit this dependence to assess correlations between spikes in neighboring VS cells and $\mathrm{V} 1$. Our results suggest that even when presynaptic graded potential shifts control the overall rate of postsynaptic spikes, the exact time of their occurrence is triggered by presynaptic spikes.

\section{EXPERIMENTAL PROCEDURES}

The preparation of the animals and the recording procedures followed exactly the methods described in Beckers et al. (2007). Young female blowflies (Calliphora vicina) were dissected to make the lobula plates of both brain hemispheres easily accessible for electrophysiological recordings. All experiments were notified to the German authorities and conformed to the animal welfare act. The number of animals used was kept to a minimum.

The V1 cell was recorded extracellularly in its output region in the left brain hemisphere with 1-5 M $\Omega$ glass electrodes filled with $2 \mathrm{M}$ potassium acetate. VS cells were recorded intracellularly at their axonal output region in the right brain hemisphere with sharp glass electrodes with 15-30 $\mathrm{M} \Omega$ resistance filled with $2 \mathrm{M}$ potassium acetate using an npi SEC-10LX amplifier (npi Electronics, Tamm, Germany) with the standard low-voltage head stage.

The voltage-clamp recordings were performed using the discontinuous single-electrode voltage-clamp method with a duty cycle of $1 / 4$ and a switching frequency of about $40 \mathrm{kHz}$. The use of high switching frequencies was advantageous to our experiments because this allowed us to reduce crosstalk from the intra- to the extracellular recording electrode in critical frequency ranges. The 

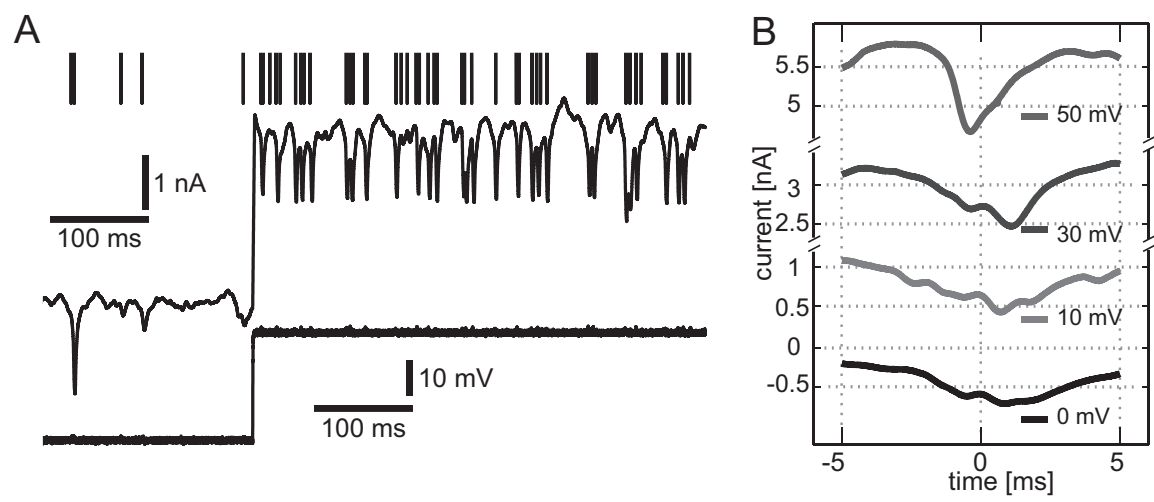

Fig. 2. Correlation between current transients in a voltage-clamped VS neuron and V1 spikes. (A) The membrane potential of a VS cell (bottom trace) is first clamped to resting potential at $-42 \mathrm{mV}$ (initial $\sim 250 \mathrm{~ms}$ shown) and then depolarized by $30 \mathrm{mV}$. Depolarization leads to increased spiking activity of $\mathrm{V} 1$ (vertical dashes, top trace). The applied holding current (middle trace) shows many transients, most of them temporally co-occurring with V1 spikes. (B) Spike-triggered average of the holding current at different depolarization levels for the same recording as shown in A. The V1 spikes were detected by setting a threshold to the extracellular signal (see Experimental procedures). The time of threshold crossing corresponds to zero on the abscissa.

waveform of the amplifier's discontinuous raw output signal was checked during each recording to tune the amplifier's filtering and gain properties to the electrode properties. In particular, care was taken that electrode time constants were sufficiently fast, i.e. that during the amplifier's voltage sampling intervals the electrode voltage had recovered from the amplifier's current pulses to a quasi steady-state that is determined exclusively by the neuronal properties.

Moving square wave gratings were used for cell identification. We used two types of custom-built LED arrays. One LED matrix consisted of $48 \times 48$ round ( $3 \mathrm{~mm}$ diameter) ultrabright blue LEDs. The LED board was obliquely aligned in a $45^{\circ}$ angle to the $0^{\circ}$ azimuth of the fly and covered a visual field from about $-10^{\circ}$ to $70^{\circ}$ horizontally and from about $-45^{\circ}$ to $25^{\circ}$ vertically. Square wave gratings with a spatial wavelength of about $25^{\circ}$ moved with a temporal frequency of 3.125 cycles/s. Horizontal and vertical pattern motion on the entire matrix or in a rectangular subarea comprising one-third or square areas of one quarter of the matrix was used for cell identification. The mean luminance as seen by the fly changed according to the viewing angle and ranged from $400 \mathrm{~cd} / \mathrm{m}^{2}\left(45^{\circ}\right.$ angle) to $5000 \mathrm{~cd} / \mathrm{m}^{2}\left(0^{\circ}\right.$ angle). The other stimulus device (used for the recording shown in Fig. 1) was a rectangular matrix of green LEDs (mean luminance $\sim 500 \mathrm{~cd} / \mathrm{m}$ ) covering a visual field from about $-20^{\circ}$ to about $45^{\circ}$ horizontally and from about +20 to $-30^{\circ}$ vertically (Beckers et al., 2007). The grating had a spatial wavelength of $17^{\circ}$ and a temporal frequency of $1.65 \mathrm{~Hz}$.

VS cells were identified in bridged recording mode by their graded depolarizations during presentation of motion in the preferred direction. As a consequence of their similar receptive field (Krapp et al., 1998) we accepted a certain degree of uncertainty in the determination; in particular we did neither distinguish between VS2 and VS3 or between VS4 and VS5. We thus, either speak generally of VS cells or use a coarse classification into VS1, VS2/3 and VS4/5 in the following.

In the voltage-clamp experiments (data shown in Figs. 2-4) we used rectangular potential steps of $600 \mathrm{~ms}$ duration as voltage commands (in some experiments all durations were prolonged to

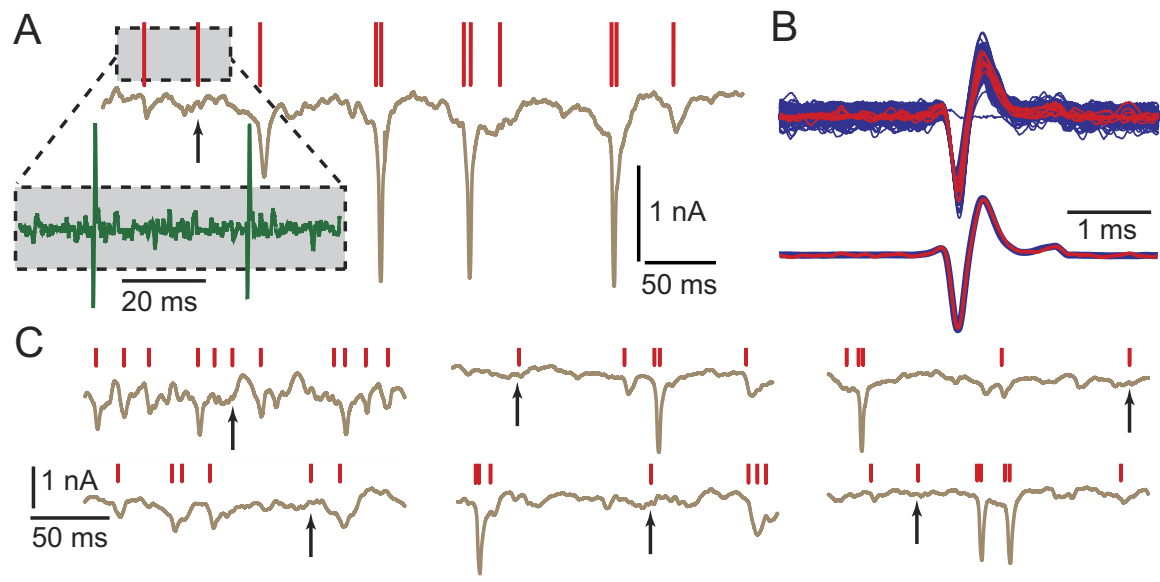

Fig. 3. Most, but not all V1 spikes are accompanied by transient currents in a voltage-clamped VS neuron. (A) Example of a failure. The upper trace (red) shows registered $\mathrm{V} 1$ spikes, the middle trace (brown) the simultaneously recorded current in a VS neuron depolarized by 20 mV (relative to the resting potential). Events where a $\mathrm{V} 1$ spike is not accompanied by a current transient (failure) are marked by arrows. The inset shows a short segment of the extracellular V1 recording. The waveforms of the two V1 spikes, the latter of which associated with a failure in the VS current trace, are similar. (B) Plot of all V1 spikes of one trial (same example as in A) to monitor the validity of the V1 spike registration. In the upper trace all spikes are plotted (blue) as well as those associated with failures in the VS current (red). The lower trace compares the averages of all V1 spike waveforms (blue, 363 spikes) with the average of the "failure" events (red, eight failure situations). (C) Several example traces from three different dual recordings in which individual V1 spikes were associated with failures in the VS current trace. All failures are marked by an arrow. 

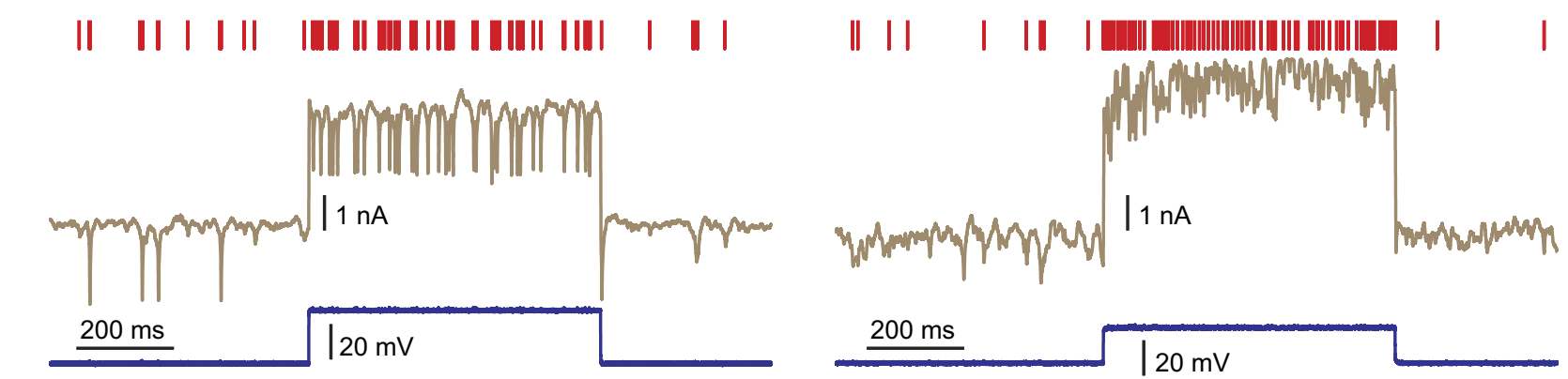

C
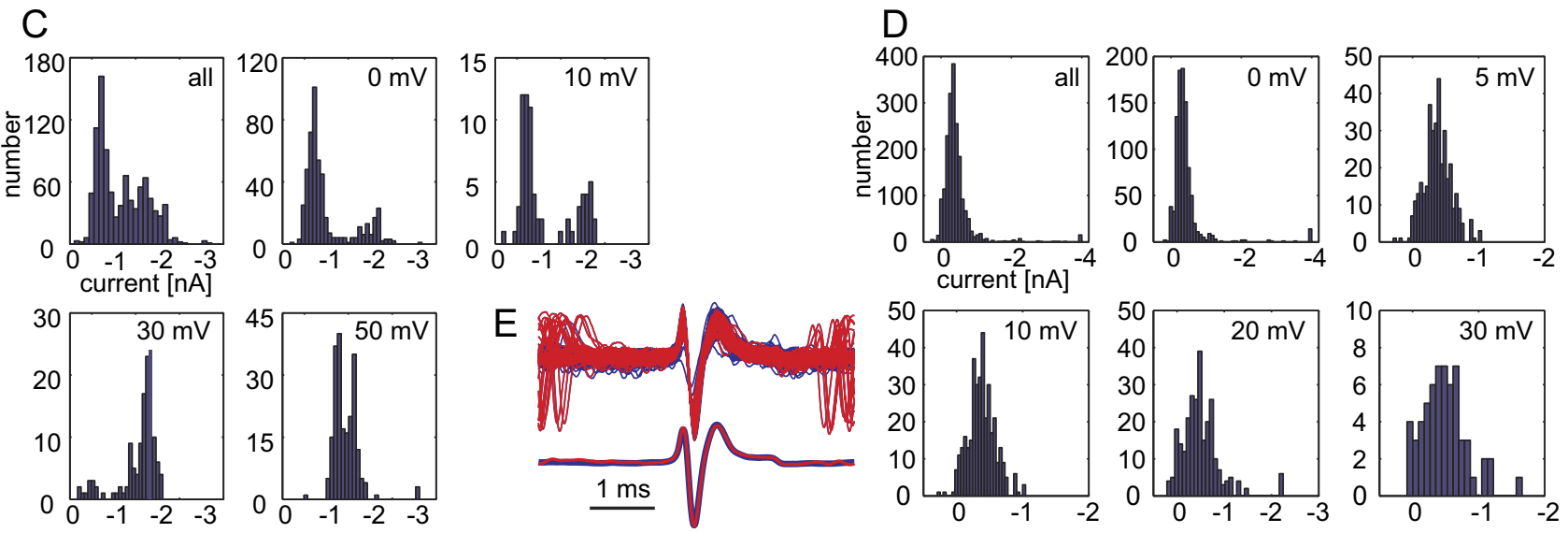

Fig. 4. Distribution of current transients in voltage-clamped VS cells associated with V1-spikes. (A, B) Example traces from two different VS-V1 cell pairs. The vertical lines in the top trace denote detected V1 spikes, the bottom trace shows the VS cell membrane potential and the middle trace shows the holding currents. In A V1 spikes co-occur either with marked current transients or with very small events (or even "failures") in the VS neuron (classified as VS2/3), whereas in B the amplitude of VS (classified as VS1) current transients rather forms a continuum. (C, D) Histograms of frequency distributions of V1-spike-associated current transients in the VS cells shown in A and B, respectively, clamped at different holding potentials relative to resting potential. Axis labeling is shown exemplarily for one example each. Top left subplots show distributions of all V1-spike-associated current transients in VS, regardless of holding potential. The other subplots show the distributions for various depolarized voltage-clamp potentials relative to the resting potential. (E) To exclude the possibility that the two classes of current transients in VS as shown in C are associated with two different types of spikes, one class being erroneously assigned to V1, extracellularly recorded spike waveforms accompanied by small (top trace, blue) or large (top trace, red) current transients are plotted. The bottom traces show the mean shape of spikes accompanied by small (blue) current transients and the mean spike shape of all spikes accompanied by large (red) current transients in VS.

$1200 \mathrm{~ms}$ ). These steps were provided in one of two protocols, one applying a sequence of voltage steps to $+5,-5,+10,-10,+20$ and $-20 \mathrm{mV}$ relative to the resting potential, and the other applying the following voltage steps: $+10,-10,+30,-30,+50$ and $-50 \mathrm{mV}$. In both protocols each step was followed by $600 \mathrm{~ms}$ (respectively $1200 \mathrm{~ms}$ ) duration of clamping the voltage at the resting potential.

Current pulses were applied in the bridge mode to elicit spikes in the recorded VS cell and to monitor spiking activity in the V1 neuron (data shown in Figs. 5 and 6). The pulses were injected at $50 \mathrm{~Hz}$ and lasted $2 \mathrm{~ms}$ each. Twenty pulses of identical amplitude were applied in a sequence, followed by $300 \mathrm{~ms}$ without current pulses. Current pulse amplitudes of 2, 2.5, 3, 3.5, 4, 4.5 and $5 \mathrm{nA}$ were used. One trial consisted of eight pulse sequences with different amplitudes arranged in a pseudo-random order. In six of these sequences the current pulses were superimposed on tonic current injection of $1 \mathrm{~s}$ duration, starting $25 \mathrm{~ms}$ prior to the first pulse. Tonic current amplitudes of $-1.5,-1,-0.5,0.5,1,1.5$ and $2 \mathrm{nA}$ were applied in pseudo-random order.

All data acquisition and stimulation were done using selfwritten software (Visual C ++6.0 , Microsoft, Redmond, WA, USA) on a standard personal computer. The raw extracellular $\mathrm{V} 1$ signal, the VS membrane potential and the applied voltage-clamp current were recorded with a high-precision data acquisition board (PD2MFS-4-500/14, United Electronic Industries, Canton, MA, USA) at a sampling frequency of $100 \mathrm{kHz}$ for each channel. V1 spikes were detected off-line by setting an appropriate threshold to the recorded extracellular signal. The time of threshold crossing was taken as the instant of spiking (e.g. for spike-triggered averages and overlay plots of multiple spikes). Spike detection was suspended in a 1-ms window after each spike to avoid multiple detection of the same spike. The same method was applied for detection of VS spikes when current pulses were applied. The VS spike detection threshold was individually determined for every trial (see Fig. 5A).

Calculation of the probabilities of binominal distributions followed the method developed by Laplace (1812), estimation of corresponding 95\% confidence intervals followed the adjusted Wald method (Wilson, 1927; Agresti and Coull, 1998).

All data analysis was performed off-line using custom analysis routines written in MatLab (The MathWorks, Natick, MA, USA).

\section{RESULTS}

Simultaneous dual recordings in a visual motion-processing region of the fly brain were performed in vivo to study synaptic signal transfer between identified neurons (recording site and motion pathway sketched in Fig. 1). We recorded from 16 VS-V1 cell pairs. In eight cell pairs the 
$\mathrm{A}_{*}$

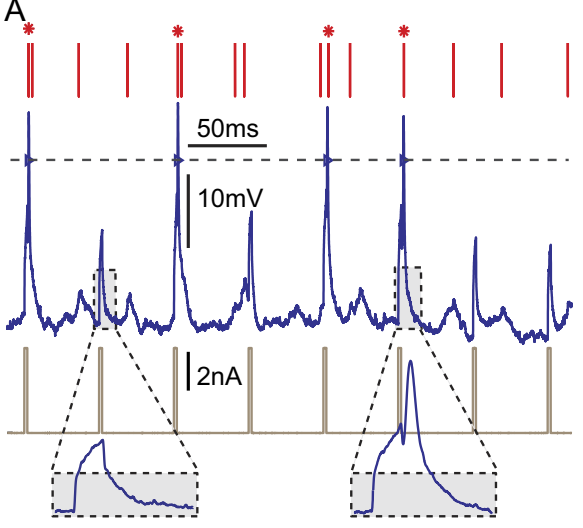

B

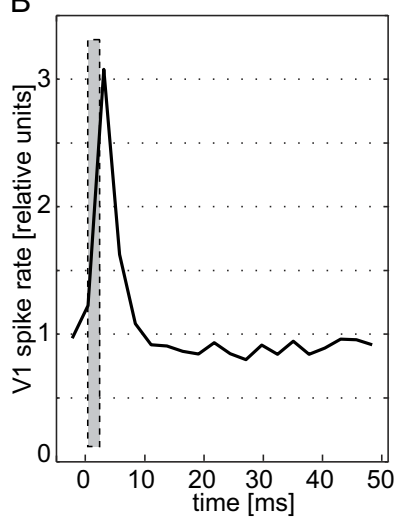

C

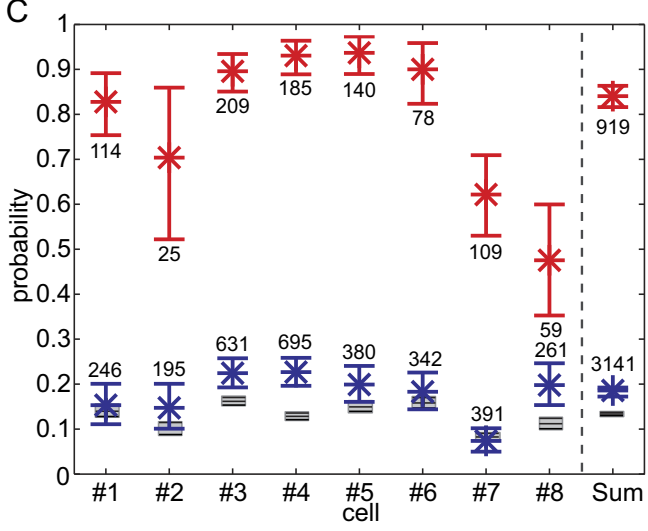

Fig. 5. VS spikes elicit with high probability V1 spikes. (A) Impact of 2 ms current pulses (bottom trace, brown) on VS cell potential (middle trace, blue) and V1 spike rate (top trace, red vertical lines. In many cases the current pulse elicits a spike in VS. To detect these events we determined whether the VS potential crossed a threshold (dashed horizontal line) in a 5-ms time window starting 1 ms before the current pulse. The same time window was also tested for the occurrence of V1 spikes; the asterisk symbols above the top trace mark V1 spikes within the 5 ms time window. The insets magnify the VS potential on a fine time scale (abscissa $10 \times$, ordinate $0.75 \times$ ) for two different current pulse dependent events. The course of the potential in the left inset represents a subthreshold response of the VS neuron to the current pulse whereas the right inset shows a spike riding on top of the passive voltage response. The threshold value was set individually for each trial after visual inspection of several single events and their classification as being active or passive. (B) Current-pulse-triggered average of the V1 spike rate. The 2-ms current pulse started at 0 ms and is indicated by the grey tinted bar. Data from eight cells were averaged. The spike rate was normalized to the average spike rate of each entire trial (see Experimental procedures). (C) Probability of $\mathrm{V} 1$ spike occurrence during the 5-ms window (see A) when either a VS spike was detected (red asterisks) or no VS spike was detected (blue asterisks), see A for VS spike detection. The bars indicate a 95\% confidence interval (adjusted Wald method); the numbers at each bar denote the number of trials evaluated. A total of eight cells has been evaluated; the right column labeled "sum" shows the probability averaged over all eight cells.

VS-cell was recorded in voltage clamp mode. Three of these pairs were assigned as VS1-V1, four as VS2/3-V1 and one as VS4/5-V1. In the other eight cell pairs current pulses were applied in bridged mode, five of them were assigned as VS1-V1 the other three as VS2/3-V1.

When visually stimulated with movement in the preferred direction (downward), the presynaptic VS cells respond with a strong axonal graded membrane depolarization of about $10-15 \mathrm{mV}$ relative to their resting membrane potential. Additionally, spikes of variable amplitude of up to $40 \mathrm{mV}$ are superimposed on the graded depolarization (Fig. 1, bottom diagram). The spike frequency may reach $60-100 \mathrm{~Hz}$. When motion in the null direction (upward) is presented, VS cells hyperpolarize by up to $-10 \mathrm{mV}$ relative to the resting level.

The $\mathrm{V} 1$ cell is a spiking neuron that receives excitatory input from several VS cells. Therefore the spike rate of V1 is raised up to values of about $250 \mathrm{~Hz}$ when the presyn-
A

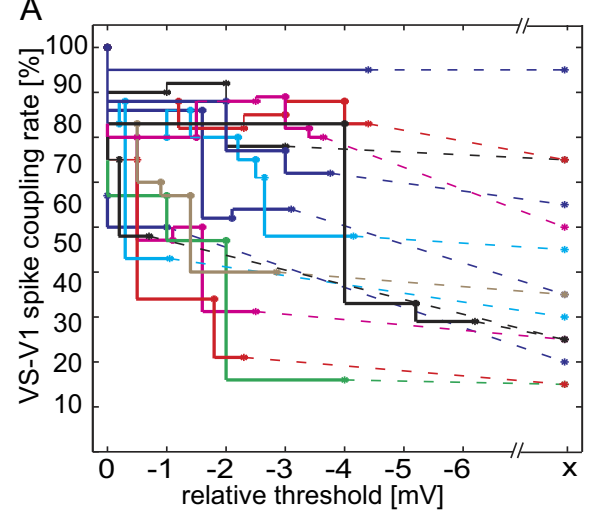

B

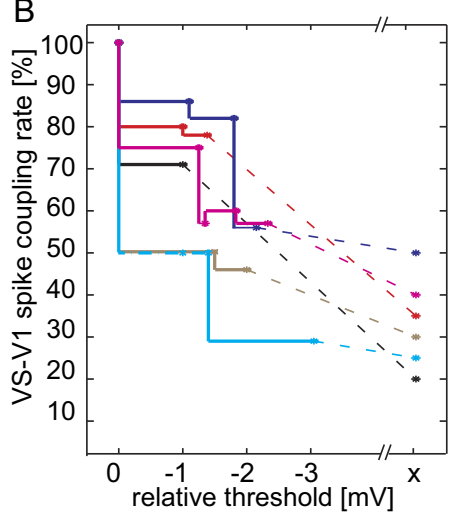

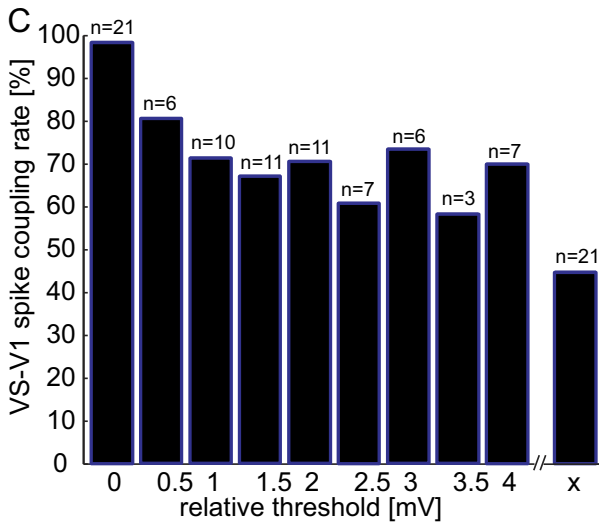

Fig. 6. VS-V1 spike coupling rate depends on VS spike amplitude. (A, B) VS spikes were elicited by current pulses and their co-occurrence with V1-spikes was determined by the same procedure as in Fig. 5. Since VS spikes differ in their amplitude (see Fig. 5A) shifting the threshold for their detection changes the proportion of events classified as VS spikes. The plots show how a shift in threshold affects the VS-V1 spike coupling rate of single trials (lines with different colors) recorded in one VS-V1 cell pair. Zero on the abscissa corresponds to a threshold that leads to detection of only those VS spikes with the largest amplitude. Accordingly, these events are accompanied by V1 spikes with a high probability and a further increase in the threshold does not lead to a higher coupling rate. The rightmost value on the abscissa, termed " $X$ " corresponds to the maximum threshold value that leads to counting every VS transient that is elicited by current pulse as a VS spike. The steps in the lines for individual trials result from the fact that more and more discrete unitary events are classified as VS spikes when gradually lowering the threshold. (A) Data for 15 trials of current pulses riding on a depolarizing tonic current; $B$ shows data from six trials with current pulses applied on resting potential. (C) Data of $A$ and $B$ were pooled to bins of $0.5 \mathrm{mV}$ width to obtain average VS-V1 spike coupling rates. The numbers on top of the bars denote the number of data points available within each bin. 
aptic VS cells are activated by motion in the preferred direction (see example recording in Fig. 1; Kurtz et al., 2001; Warzecha et al., 2003). Without motion, V1 has a spontaneous spike rate of about $20-30 \mathrm{~Hz}$. Motion in null direction can entirely block spike generation in V1 (see Fig. 1).

\section{Coincidence of pre- and postsynaptic spikes in the VS-V1 circuit}

Whenever a VS spike occurs a V1 spike is generated with a high probability within a narrow time window (Kurtz et al., 2001; Warzecha et al., 2003). However, since visual stimulation alone was used in these studies to activate the neurons, several aspects remained unresolved concerning synaptic transfer of fast signals: First, it was unclear whether spikes in V1 were elicited by spikes in the recorded VS neuron or in one of the neighboring neurons. This uncertainty exists because the spatial receptive fields of neighboring VS neurons overlap to a large extent (Hengstenberg et al., 1982; Krapp et al., 1998) and thus, visual stimulation cannot be confined to a single VS neuron. Second, it remained unclear in how far graded depolarization and spikes of VS neurons interact to control spike rate and spike timing of $\mathrm{V} 1$. The role of graded potential changes and spikes could not be disentangled because visual stimulation produced a mixture of both signals in the VS neurons with depolarization strength and spike frequency highly correlated. To some extent, this problem has been tackled in a former study by voltage-clamping single VS neurons (Beckers et al., 2007). There it was shown that constant depolarization of a single VS cell is sufficient to increase the $\mathrm{V} 1$ spike rate to a high level and to maintain this level over several hundred milliseconds. Although these results show that presynaptic spikes are not an indispensable prerequisite to elicit postsynaptic spikes, it remained open whether the timing of postsynaptic spikes is controlled by the occurrence of presynaptic spikes. More specifically, the question arises whether V1 spikes are triggered by VS spikes or whether the temporal spike correlation between VS and V1 is due to a mutual interaction via an electrical coupling between VS and $\mathrm{V} 1$. To track down the origin of the VS-V1 spike co-occurrence, we closely inspected the voltage clamp holding currents applied to VS neurons during VS-V1 dual recordings. In particular, we paid attention to the correlation of transients in the holding currents with the occurrence of V1 spikes under various conditions.

When a VS cell is voltage clamped the currents required to maintain the command potential reflect not only intrinsic properties of the recorded cell, but also the inputs of the cell from synaptically coupled neurons. On a coarse time scale the injected holding current remained fairly constant on a level depending on command voltage (see Fig. 2A). However, many additional pronounced brief current transients reminiscent of excitatory postsynaptic currents occurred. Even upon gross inspection these current transients appear to coincide with postsynaptic V1 spikes. Correlation between current transients in the voltageclamped VS neuron and V1 spikes was present in all cells irrespective of the clamp potential (see Fig. 2B), i.e. both during low and high spiking activity of the V1 cell.

Since no motion stimulus is present, only two scenarios can explain the co-occurrence of current transients in the voltage-clamped VS cell with V1 spikes: First, the clamped VS neuron and V1 might receive a common excitatory input. Plausible candidate neurons delivering such a common input are the neighboring VS cells, because electrical coupling between neighboring VS cells has been shown to cause temporal correlation of voltage transients (Haag and Borst, 2004). Second, there might be a reciprocal interaction between the VS neuron and V1, potentially also via an electrical synapse. These alternatives will be distinguished by the following analysis.

\section{Spikes in V1 coincide in most but not in all cases with prominent current transients in voltage-clamped VS neurons}

Although it is not yet known whether VS and V1 are coupled by electrical or chemical synapses exclusively or by mixed electrical and chemical connections the impact of photoablation of single VS neurons on V1 activity (Kalb et al., 2006) is best compatible with an electrical synapse connecting at least one of the VS cells, i.e. VS1, to V1, but chemical synapses connecting the other VS cells to V1. The current transients in voltage-clamped VS cells coinciding with V1 spikes can help identify an electrical synapse between the recorded VS cell and the V1 cell: If the synaptic connection were electrical, every $\mathrm{V} 1$ spike would lead to a current transient in the voltage-clamped VS cell. Moreover, fairly uniform amplitudes of these transients would be expected, since neither large variation in input resistance of clamped VS cells in the absence of visual stimulation, nor large heterogeneity of the amplitudes of V1 spikes is probable.

We monitored the currents during voltage clamp of a VS2/3 neuron and found rare events when V1 spikes were not accompanied by a prominent (see below) current transient in the clamped VS cell. These events are termed "failures" throughout the following. In Fig. 3A/C examples of V1 spikes and the injected holding current in VS are shown: a $\mathrm{V} 1$ spike may be accompanied by a large-amplitude transient current in the voltage-clamped VS cell in one case, whereas the current trace remains entirely flat during the occurrence of another V1 spike. Since current injections into VS were fluctuating, it is not always easy to assess whether a V1 spike is accompanied by a current transient in VS. For a quantitative analysis we detected the minimum of current amplitudes within a time window of 4.5 ms width starting $2 \mathrm{~ms}$ before the corresponding V1 spike relative to a baseline value obtained from averaging in a 12 ms window starting $8 \mathrm{~ms}$ before the corresponding $\mathrm{V} 1$ spike. Current transient amplitudes $\geq$ zero were counted as failures. With this measure, on average $3.1 \% \pm 1.8$ of $\mathrm{V} 1$ spikes (mean over eight cells \pm standard deviation, total number of registered V1 spikes: 15,504) were not accompanied by a negative current transient in the VS cell and thus classified as failures. Additionally the detected failures were verified by visual inspection of the current traces. 
Although visual inspection is dependent on subjective decisions it led to a similar estimation of failure rates as the automated detection. To exclude the possibility that the detection of V1 spikes was not correct in the case of failures of VS current transients, we also subjected the extracellular recording traces to a close inspection in these cases. There was no indication of false-positive detections of V1 spikes in the case of VS failures. This can be clearly seen for the example shown in Fig. $3 \mathrm{~A}$ and the analysis shown in Fig. 3B where the extracellularly recorded waveforms of all V1 spikes detected in a recording trial and the waveforms of all V1 spikes that accompanied failures of the VS current are plotted in blue and red, respectively. The waveforms of both classes are very similar, as is also the case for the respective mean traces (see Fig. 3B, bottom).

\section{V1-spike-associated current transients in voltage-clamped VS neurons are variable and often form distinct amplitude classes}

Although the presence of "failures" is good evidence against a substantial electrical coupling between V1 and the recorded VS neuron, the scarcity of "failures" prohibits strong conclusions. Therefore, we analyzed in more detail the amplitudes of the current transients in voltage-clamped VS neurons. Fig. 4A and $B$ shows short segments of dual recordings of two different cell pairs during which the holding potential of the VS neuron was changed from resting potential to a depolarized level. The variability in the amplitudes of current transients in VS that are associated with V1 spikes appears to differ between the two cell pairs: in the VS2/3 neuron shown in Fig. 4A the current transients are very heterogeneous, either forming very clearly defined large deflections or small, sometimes hardly visible events. In contrast, the current transients in the VS1 neuron shown in Fig. 4B seem to be more evenly distributed and generally smaller in amplitude. To analyze the amplitude distribution of current transients quantitatively, we determined for every detected V1 spike the amplitude of the VS current transient as described in the section above. Fig. 4C shows for the VS2/3-V1 cell pair the frequency distributions of current amplitudes binned into different classes. As is most clearly visible in the frequency distribution summed over all events, disregarding potential differences in the frequency distribution for different holding potentials applied to the VS neuron (see below), the distribution is bimodal, with one peak at low amplitudes (about $0.5 \mathrm{nA}$ ) and a second peak at a much higher amplitude (about $2 \mathrm{nA}$ ). The presence of two classes of current transients in VS indicates that V1 spikes are associated with two distinct kinds of excitatory input to the VS cell. Rare events previously classified as "failures" cannot be distinguished from the low-amplitude class of inputs in the frequency histogram analysis.

Distinct classes of small and large current transients associated with V1 spikes were not present in the VS1 neuron (compare VS2/3-V1 cell pair shown in Fig. 4A, C with the VS1-V1 cell pair shown in Fig. 4B, D). In the VS1 cell the current transients were distributed over a continu- ous range while in others a clear separation between classes of small and large current amplitudes exists. In six out of eight recorded cell pairs the VS current transients were distributed over a rather continuous range while in two cells a clear bimodal distribution was observed. Thus the different amplitude distributions of the VS current transients may result from a difference in the synaptic connection to V1, with VS1 forming predominantly electrical synapses with V1 and VS2/3 forming predominantly chemical synapses, in accordance with Kalb et al. (2006). However, bimodal versus unimodal distributions of current amplitudes were not consistently related to the types of VS neurons. Both neurons that showed a bimodal distribution were classified as VS2/3, but among the neurons that showed an unimodal distribution three were classified as VS1, two as VS2/3 and one as VS4/5.

The large variability and, in particular, the different classes of inputs are hardly compatible with the concept that transient current inputs into the voltage-clamped VS cell are due to electrical coupling of VS and V1 and are present whenever $\mathrm{V} 1$ fires a spike, elicited for example by activity in other VS cells. We propose that the coincidence of current transients in VS cells and V1 spikes and the large variability in current amplitudes originates mainly for another reason: VS cells were shown to be coupled to neighboring VS cells in a chain-like manner by electrical synapses and voltage transients were demonstrated to co-occur in neighboring VS cells (Haag and Borst, 2004). This makes it plausible that a current transient in a VS neuron is elicited whenever a spike is present in one of the neighboring VS neurons. Unlike direct electrical coupling between the recorded VS neuron and V1, the presence of different amplitude classes of current transients can then easily be explained if the current transients were regarded as being caused by spikes in neighboring VS neurons: since all VS cells but VS1 have two direct neighbors, differences in the coupling strength with its neighbors could lead to different amplitudes of current transients in the recorded VS neuron. Moreover, unlike V1 spikes the spikes of graded-potential VS neurons vary in their amplitudes (Hengstenberg, 1977; Haag and Borst, 1998). This property could also contribute to the variability in the current transients in the recorded VS neuron. A third reason for variable current amplitudes and, in particular, for the presence of very small current transients is spike activity in a next-but-direct-neighbor VS cell. Failures in VS current transients could result from spontaneously elicited spikes in V1, or spikes elicited by other input than VS, e.g. inputs mediated indirectly via descending neurons from ocellar interneurons (Parsons et al., 2006).

\section{V1 spikes are triggered by VS spikes in an amplitude-dependent way}

To assess directly in which way the occurrence and timing of spikes in V1 are affected by spikes in one of the VS neurons we monitored $\mathrm{V} 1$ spiking activity during injection of brief current pulses into a VS neuron. The amplitude of these current pulses was set to a level that elicited a VS spike only for part of the current injections, whereas the 
voltage transients remained subthreshold in others. Thus, we were able to compare how efficiently spikes in V1 are elicited by brief subthreshold depolarizations of VS, on the one hand, and by VS spikes on the other hand. Moreover, a potential relevance of VS spike amplitude for V1 activity could be analyzed. This was possible because, similar to what was found during visual stimulation (Hengstenberg, 1977; Warzecha et al., 2003), VS spikes that were elicited by current injection were not all-or-none, but graded in their amplitude.

Based on the inspection of the time courses of the pulse-evoked voltage transients in VS neurons a threshold was set that separated VS spikes from subthreshold voltage transients as best as possible. By this simple procedure some of the VS spikes might be counted as subthreshold depolarizations or vice versa. This difficulty is, however, not critical for the conclusions to be drawn in the following. In contrast, it highlights the principal point that, as a consequence of the graded nature of VS spike signals, voltage transients in VS form a continuum rather than two distinct types of events, namely purely passive depolarizations and full-blown action potentials. In Fig. 5A examples of events are shown that were classified by the threshold (indicated by the dashed line) as VS spikes, on the one hand, or as subthreshold depolarizations, on the other. We analyzed quantitatively in a sample of VS cells $(n=8)$ how efficiently these two classes of events trigger a V1 spike within a narrow time interval. As can be seen in Fig. 5B the events classified as VS spikes trigger a V1 spike (red asterisks) with a much higher probability than the events classified as subthreshold depolarizations (blue asterisks). This difference was very consistent for all cell pairs and it changed in a quantitative way but not qualitatively when shifting the threshold value for the classification of the pulse-evoked depolarizations. The consequence of the chosen threshold value is made more explicit in Fig. 6, which plots the probability with which VS voltage transients exceeding a given threshold elicit a V1 spike. Raising the threshold and, thus, selecting only large VS spikes, increased the proportion of events that elicit a V1 spike (see values on left side of abscissas of Fig. 6). The above findings corroborate the graded nature of VS-V1 synapses by showing that large VS spikes are more effective in eliciting a spike in the postsynaptic V1 neuron than small VS spikes or subthreshold depolarizations. A second finding is that brief, subthreshold depolarizations in VS, although much less effective than VS spikes in eliciting V1 spikes, do still have an impact on V1 spiking. This conclusion can be made when comparing the probability with which these events elicit V1 spikes with the corresponding probability of finding a $\mathrm{V} 1$ spike in an identically sized time window in the absence of current pulse injection into VS (Fig. 5B; compare blue asterisks with grey bars). This finding is compatible with the previous observation that voltage clamp of a VS cell to a sustained depolarized voltage level increases $\mathrm{V} 1$ spiking in a graded manner (Beckers et al., 2007).

\section{Synchronization in the presynaptic cell ensemble is} mediated by fast signals via electrical synapses

The association of current transients in VS with V1 spikes (see Fig. 2B) and their explanation as being mainly caused by spikes in neighboring VS neurons indicate one important feature of fast signaling in the VS-V1 circuit: Spikes appear to be synchronized not only between one VS neuron and the V1 neuron but often also between several VS neurons and V1. The presence of large amplitude current transients in the recorded VS neurons suggests they would often also spike in synchrony with V1 and neighboring VS neurons if they were not clamped to a fixed membrane potential.

To assess the role of spiking synchronization among VS neurons we determined the impact of voltage clamping one presynaptic VS cell to its resting potential on spike activity of the V1 neuron. This experiment was performed without visual stimulation to avoid shared input of the VS cells, except for upstream noise sources. The recording condition was alternated between bridged mode and voltage-clamp to resting potential. The mean spike rate of $\mathrm{V} 1$ was $24.8 \mathrm{~Hz}$ when the VS cell was not clamped and 24.3 $\mathrm{Hz}$ when the VS cell was clamped. Thus, clamping one VS cell to its resting potential did not obviously influence the spontaneous spike rate of $\mathrm{V} 1$. However, the analysis of the interspike intervals revealed a change in the temporal distribution of the V1 spikes. In the unaffected situation spikes often occur in spike doublets. Here we refer to doublets as sequences of two spikes within less than $3 \mathrm{~ms}$. The presence of doublets becomes evident as a disproportionately large lowest class in the frequency distribution of interspike intervals (Fig. 7). When one VS cell was clamped, the doublet rate dropped from $17.3 \%$ (out of 594 interspike intervals) in the non-clamped situation to $10.1 \%$ doublets (out of 584 interspike intervals) while being clamped (compare Fig. 7). This drop in doublet rate with voltage-clamp to resting potential is significant because the $95 \%$ confidence interval calculated according to the adjusted Wald method (Wilson, 1927; Agresti and Coull, 1998) for the two conditions does not overlap (bridged: $14.3 \%-20.4 \%$; voltage clamp: $7.8 \%-12.6 \%$ ). This finding supports the hypothesis that electrical coupling between the VS cells plays a role in synchronizing activity in the VS-V1 network. Spike doublets in V1 with very small interspike intervals are indicative of synchronized activity in the presynaptic VS neurons: A spontaneous spike by one VS cell may elicit after a short delay a spike in a neighboring VS cell, and this synchronized VS cell activity has a high potential to trigger two V1 spikes in close sequence. When clamping one VS cell to its resting value, it is no longer able to become synchronized with its neighbors and thus the $\mathrm{V} 1$ cell receives fewer synchronized inputs.

\section{DISCUSSION}

While action potentials are required to convey information along long axons, many sensory neurons and local interneurons convey information via graded de- and hyperpolarizations or by a mixture of graded signals and action potentials. In this study we addressed the significance of 

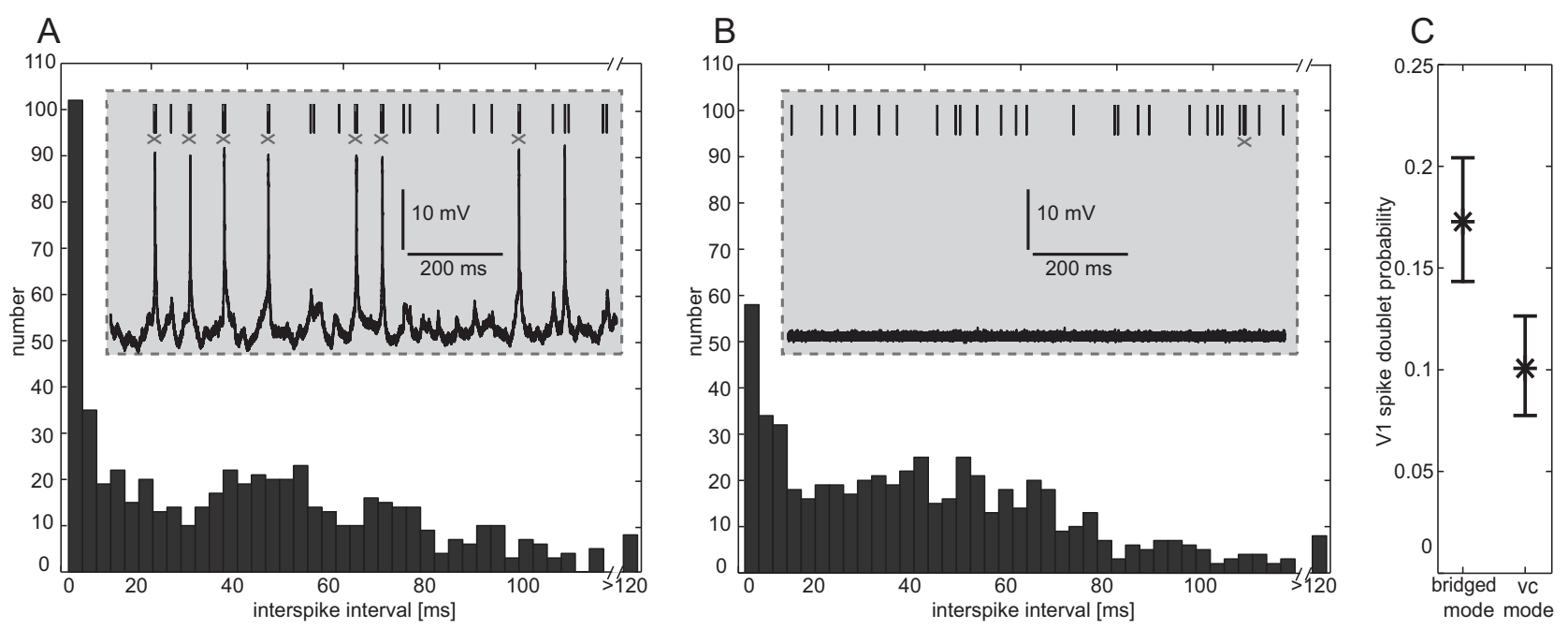

Fig. 7. Impact of voltage clamping one VS neuron to its resting potential on interspike interval distribution of $\mathrm{V} 1$. The histograms show the frequency distributions of the temporal distance between two V1 spikes when one VS neuron was recorded in bridge mode (A) or voltage-clamped at its resting potential at $-53.5 \mathrm{mV}(\mathrm{B})$. Both plots show data of the same cell, with several trials for each condition recorded in alternation. The spike rate is virtually the same in both approaches ( 24.8 vs. $24.3 \mathrm{~Hz}$ ). Thus, similar number of spikes were recorded in bridge mode $(n=594)$ and in voltage-clamp mode $(n=584)$ and histogram data were not normalized. The insets show example traces of both recording conditions with registered $\mathrm{V} 1$ spikes (vertical lines) shown on top and VS membrane potential shown in the bottom trace. Doublet spikes were detected as two spikes occurring with a temporal distance of less than $3 \mathrm{~ms}$, corresponding to the first column of the histogram, and are marked by a cross symbol. (C) Proportion of spike doublets in bridged mode and with voltage clamp to resting potential. Error bars denote confidence intervals.

these different forms of electrical signals for the timing of spikes in a neuronal circuit of motion sensitive neurons in the blowfly brain.

When injecting brief current pulses in one of the VS neurons, spikes in the postsynaptic $\mathrm{V} 1$ neuron were more reliably evoked in those cases when the current pulse reached threshold for spike initiation in the VS neuron. Nonetheless, subthreshold current injections raised the probability of $\mathrm{V} 1$ spiking above its spontaneous level. This result, together with our finding that the probability of $\mathrm{V} 1$ spiking was dependent on the amplitude of VS spikes, suggests a dual control of V1 spiking by presynaptic graded and spike signals. Our results are, however not an entirely conclusive evidence for such a dual control, because subthreshold events might elicit spikes in neighboring presynaptic VS neurons, which have been found to be coupled in a chain-like fashion by electrical synapses (Haag and Borst, 2004, Fig. 1). Spikes in neighboring VS neurons might then in turn elicit spikes in V1. We consider this scenario not very likely, because the coupling strength of VS-VS electrical connections is weak, as characterized by low coupling coefficients of less than 0.1 (Haag and Borst, 2004). Thus it is improbable that current pulses that do not even elicit spikes in the injected cell are strong enough to elicit a spike in a neighboring VS neuron.

We controlled the graded input to the postsynaptic V1 neuron via voltage clamp of one of the presynaptic VS neurons and simultaneously registered the postsynaptic spike activity as well as the transients in the presynaptic holding current. We interpreted these current transients as being indicative of spikes in the neighboring VS neurons that are electrically coupled with the recorded cell. Since temporal co-occurrence of current transients and postsynaptic spikes was maintained not only at resting potential but also during strong tonic depolarization of one presynaptic neuron, we conclude that presynaptic spikes can control the precise timing of postsynaptic spikes even in the presence of strong graded input.

\section{Origin of current transients in voltage-clamped VS neurons}

In the analyzed VS-V1 circuit it is difficult to gain certainty about the origin of the current transients in voltageclamped VS neurons, since it has not yet been entirely clarified whether VS-V1 synapses are electrical or chemical or are mixed electrical and chemical. If they were purely electrical, current transients in a voltage-clamped VS neuron could result directly from the $V 1$ neuron, at least when the electrical synapses transmit in a bidirectional way. Bidirectionality appears to be characteristic of most analyzed electrical synapses (but see, e.g. for exceptions: Furshpan and Potter, 1959; Auerbach and Bennett, 1969). We provided evidence against strong bidirectionally transmitting electrical synapses between the recorded VS neuron and V1. Only some V1 spikes were associated with large-amplitude current transients in VS, whereas a large portion of $\mathrm{V} 1$ spikes was associated with only small or even undetectable current transients in VS. Even if the small currents were the consequence of a direct electrical coupling between V1 and VS (and the "failures" due to noise in the current recording), the electrical coupling between V1 and VS would be very weak, with a coupling coefficient even lower than that between neighboring VS neurons (Haag and Borst, 2004). We therefore conclude, that the synaptic coupling between the recorded VS neurons and V1 (see strong effect of depolarization of VS on $\mathrm{V} 1$ spike rate in Figs. 2 and $4 \mathrm{~A}$ ) has at least a strong 
chemical component or is the consequence of a rectifying electrical synaptic interaction.

In an attempt to clarify whether VS-V1 synapses are electrical or chemical we replaced during dual recordings insect Ringer solution with a solution containing a high concentration of the calcium-channel blocker cobalt (data not shown). Although cobalt has been demonstrated to block calcium currents in fly visual motion-sensitive neurons (Haag et al., 1997) we observed a gradual decline of the responses of VS and V1 to visual motion only after very long durations and not in all recordings. This renders an unequivocal interpretation of these results difficult. Nevertheless, in those recordings in which an effect of cobalt on the activity of the $\mathrm{V} 1$ neuron was observed, it was similarly strong during either visual stimulation or current injection into the presynaptic VS neuron. This result speaks against strong electrical coupling between VS and V1 because in this case excitation of V1 via current injection into VS should remain unaffected by cobalt. This should be the case even when the response to visual stimulation, which is dependent on chemical synapses in the periphery, is blocked.

We observed differences between VS neurons with respect to the amplitude distribution of current transients associated with V1-spikes. This heterogeneity might reflect a difference in the type of synapse between individual VS neurons and V1, in accordance with Kalb et al. (2006) who suggested electrical synapses between VS1 and V1 and chemical synapses between the other VS neurons and V1. Nonetheless, we regard it improbable that any of the neurons recorded here was coupled to V1 exclusively by electrical synapses, because small-amplitude current transients were present in any case, regardless of whether the amplitude histogram was bimodal or not. In general, our results support the idea that direct electrical coupling between V1 and VS is at most weak and leads, if at all, to small current transients in VS. To this small current component a larger component is added whenever a neighboring VS neuron is active in synchrony with the V1 neuron. The amplitudes of the latter components can either fall into distinct classes or form a continuum, depending on the contributing VS cell (nearest neighbors or more distant ones) and on the variability of spike amplitudes in neighboring VS cells.

In principle, current transients in a VS neuron with large amplitude variability could also be interpreted as being directly caused by spikes in an electrically coupled $\mathrm{V} 1$, given that the spike initiation zone in V1 shifts its position in the dendrite. If large enough, such shifts in spike initiation could potentially lead to marked changes in electrotonic distance between the spike zone in V1 and the recorded VS neuron. We regard this possibility unlikely, in particular because amplitude variability in VS current transients was present even for V1 spikes in close temporal proximity during conditions in which synaptic input to $\mathrm{V} 1$ was dominated by the voltage-clamp command to the recorded VS neuron. If at all, shifts in spike zone might be more probable when $\mathrm{V} 1$ receives visual input from variable members of the ensemble of presynaptic neurons, which might synapse on different parts of the dendrite.
Improper clamping of the VS neurons might be an alternative origin of current transients. As a consequence, the current transients may be due to spikes generated in other parts of the recorded neuron. We do not claim that fine arborizations of the dendrite, which are distant from the recording electrode, attain the holding potential. In a detailed compartmental model of VS neurons, the effect of strong axonal depolarizing current injections was found to drop to about $40 \%$ in fine dendritic tips (Haag et al., 1997). However, space clamp in the model was much better in the region of the synaptic terminal, which is closer to the electrode and less arborized. Spike generation near the terminal region is, therefore, unlikely, and spikes elicited in the dendrite would stay local and not be able to pass the recording site to the terminal. Moreover, we regard it in general very implausible that spikes are generated in the recorded neuron when it is voltage-clamped to a depolarized level, because spike initiation in VS neurons has been found to be enhanced by hyperpolarizing current injection and to be effectively blocked by sustained depolarizing current injection (and the more so should voltage-clamping), which tonically inactivates voltage-activated sodium channels (Hengstenberg, 1977; Haag and Borst, 1998). Exactly the opposite happened in the present study with the large-amplitude current transients in voltage-clamped VS neurons: their frequency increased with voltage-clamp depolarization and decreased with hyperpolarization. This result is incompatible with spiking of the recorded neuron itself, but easily compatible with spiking of neighboring VS neurons. An increased spike frequency of neighboring neurons during depolarization of the recorded neuron is plausible because of their weak electrical coupling. This coupling would cause a slight depolarization of the neighboring neuron, raising voltage fluctuations that are normally subthreshold above the threshold for spike generation.

After submission of a revised version of our paper Haag and Borst (2008) published a study, in which electrical synapses between VS1 and V1 were directly demonstrated by simultaneous intracellular recording from this cell pair. This result corroborates the wiring scheme considered most plausible by Kalb et al. (2006) and it is in general accordance with the results presented in the present study, although we conclude that chemical and electrical coupling probably coexist in the connection between VS1 and V1. The synapses between other VS neurons and $\mathrm{V} 1$ were also concluded to be electrical by Haag and Borst (2008) because V1-spike-triggered averages of the membrane potential appeared similar for all VS neurons. In our present study, we monitor V1-spike-triggered current transients in voltage-clamped VS neurons instead of membrane potential responses and, in particular, we analyzed single events instead of averages. As outlined above, chemical synaptic transmission must be assumed to explain the properties of these events.

\section{Possible roles of spike synchronization for signal transmission}

Electrical coupling among VS neurons enlarges their receptive fields (Haag and Borst, 2004). One functional ben- 
efit of electrical coupling was shown in a modeling study to lie in a more robust representation of large-field rotatory optic flow (Cuntz et al., 2007). These studies emphasized the role of transfer of slow signals between VS neurons. It has not been analyzed so far whether synchronization of fast signals between VS neurons is present during visual stimulation and whether it is functionally relevant. The present study suggests that spike synchronization between VS neurons plays a role. As outlined above, fast current transients in voltage-clamped VS neurons most probably arise from spikes in neighboring VS neurons. Without voltage clamp many of these current transients would probably lead to spikes in the recorded VS neuron. This prediction is corroborated by our finding, that voltage clamp of one VS neuron to its resting potential reduces the number of spike doublets in the postsynaptic V1 cell. These spike doublets are probably indicative of nearly synchronous spikes in two VS neurons. Thus, fast signals appear to be synchronized among neighboring VS neurons as well as between VS and V1.

Nonlinearities that facilitate transmission of fast signals and suppression of noise in purely spike-mediated transmission, for example a high voltage threshold of presynaptic calcium channels are not useful at graded synapses, because these mechanisms would hamper the transmission of the graded signal component. This constraint complicates a reliable transfer of spike signals at synapses that also need to transmit graded signals. An electrical coupling between presynaptic neurons and the resulting synchronization of presynaptic fast signals could alleviate this problem. By acting in concert the presynaptic neurons could reliably transfer fast signals to their postsynaptic target even when their mixed potential synapses lack the nonlinear mechanisms that increase reliability of purely spikemediated transmission.

In addition, a faithful transfer of signals that differ considerably both in dynamics and in amplitude might be facilitated by a mixed electro-chemical nature of the synapse. Such a share of function at mixed electro-chemical synapses, with fast components being transferred via gap junctions and only slower components remaining when the electrical component is abolished, has been demonstrated in the shaking $B^{2}$ mutant Drosophila, which suffers from a defective gap junction protein (Thomas and Wyman, 1984; Trimarchi and Murphey, 1997; Allen and Murphey, 2007). The present study suggests that spike-mediated and graded signal transfer interact in an intricate way, in which presynaptic spikes are able to define the timing of postsynaptic spikes even when their rate is largely controlled by graded presynaptic input.

\section{Functional significance of graded and spike signals in fly motion vision}

The capacity of synapses in the investigated neuronal circuit to maintain precise timing of spikes at various levels of background activity implies a functional relevance of timing precision with respect to the coding of visual motion stimuli. This issue is currently a matter of debate. On the one hand processing of visual motion is a fairly slow pro- cess per se. Accordingly, fly motion sensitive neurons seem to encode mainly the fairly slow modulations of a motion stimulus of less than $50 \mathrm{~Hz}$ (Haag et al., 1997; Warzecha et al., 1998; Warzecha et al., 2003). Consistent with this conclusion, the modulations of ego motion that are effectively represented in the visual motion responses of these neurons cover a similar range of frequencies (van Hateren et al., 2005; Kern et al., 2005; Karmeier et al., 2006). On the other hand, although most spikes elicited by white noise velocity fluctuations were only weakly coupled to the motion stimulus, certain aspects of a stimulus, for example a brisk motion onset or direction reversal, can lead to a surprisingly precise timing of the first spike and the trial-to-trial variance of subsequent spikes might still remain fairly low due to fixed refractory periods (Warzecha et al., 2003; Nemenman et al., 2008). Thus, the maintenance of precise spike timing across synapses even in the presence of variable strengths of ongoing graded signal transfer might form a functionally relevant constraint in fly motion vision.

\section{REFERENCES}

Agresti A, Coull B (1998) Approximate is better than "exact" for interval estimation of binomial proportions. Am Stat 52:119-126.

Alle H, Geiger JR (2006) Combined analog and action potential coding in hippocampal mossy fibers. Science 311:1290-1293.

Allen MJ, Murphey RK (2007) The chemical component of the mixed GF-TTMn synapse in Drosophila melanogaster uses acetylcholine as its neurotransmitter. Eur J Neurosci 26:439-445.

Arbas EA, Calabrese I (1987) Slow oscillations of membrane potential in interneurons that control heartbeat in the medicinal leech. J Neurosci 12:3953-3960.

Auerbach AA, Bennett MVL (1969) A rectifying electrotonic synapse in the central nervous system of a vertebrate. J Gen Physiol 53: 211-237.

Awatramani GB, Price GD, Trussell LO (2005) Modulation of transmitter release by presynaptic resting potential and background calcium levels. Neuron 48:109-121.

Beckers U, Egelhaaf M, Kurtz R (2007) Synapses in the fly motion pathway: evidence for a broad range of signal amplitudes and dynamics. J Neurophysiol 97:2032-2041.

Bollmann JH, Sakmann B, Borst JG (2000) Calcium sensitivity of glutamate release in a calyx-type terminal. Science 289:953-957.

Borst A, Egelhaaf M (1989) Principles of visual motion detection. Trends Neurosci 12:297-306.

Borst A, Sakmann (1998) Facilitation of presynaptic calcium currents in the rat brainstem. J Physiol 513:149-155.

Cuntz H, Haag J, Forstner F, Segev I, Borst A (2007) Robust coding of flow-field parameters by axoaxonal gap junctions between fly visual interneurons. Proc Natl Acad Sci U S A 104:10229-10233.

De Polavieja GG, Harsch A, Kleppe I, Robinson HP, Juusola M (2005) Stimulus history reliably shapes action potential waveforms of cortical neurons. J Neurosci 25:5657-5665.

De Ruyter van Steveninck RR, Laughlin SB (1996) The rate of information transfer at graded-potential synapses. Nature 379:642645.

Dodge FA, Rahamimoff R (1967) Co-operative action of calcium ions in transmitter release at the neuromuscular junction. J Physiol 193:419-432.

Egelhaaf M, Borst A (1989) Transient and steady-state response properties of movement detectors. J Opt Soc Am 6:116-127.

Furshpan EJ, Potter DD (1959) Transmission at the giant motor synapses of the crayfish. J Physiol Lond 145:289-325. 
Graubard K, Raper J, Hartline DK (1980) Graded synaptic transmission between spiking neurons. Proc Natl Acad Sci U S A 77: 3733-3735

Haag J, Borst A (1997) Encoding of visual motion information and reliability in spiking and graded potential neurons. J Neurosci 17:4809-4819.

Haag J, Borst A (1998) Active membrane properties and signal encoding in graded potential neurons. J Neurosci 18:7972-7986.

Haag J, Borst A (2004) Neural mechanism underlying complex receptive field properties of motion-sensitive interneurons. Nat Neurosci 7:628-634.

Haag J, Bost A (2008) Electrical coupling of lobula plate tangential cells to a heterolateral motion-sensitive neuron in the fly. J Neurosci 28:14435-14442.

Haag J, Theunissen F, Borst A (1997) The intrinsic electrophysiological characteristics of fly lobula plate cells. II. Active membrane properties. J Comput Neurosci 18:7972-7986.

Hausen K (1976) Functional characterization and anatomical identification of motion sensitive neurons in the lobula plate of the blowfly Calliphora erythrocepahala. Naturforsch Z 31c:629-633.

Hausen K, Wolburg-Buchholz W, Ribi WA (1980) The synaptic organization of visual interneurons in the lobula complex of flies. A light and electron microscopical study using silver-intensified cobaltimpregnations. Cell Tissue Res 208:371-387.

Hengstenberg R (1977) Spike responses of "nonspiking" visual interneurone. Nature 270:338-340.

Hengstenberg R, Hausen K, Hengstenberg B (1982) The number and structure of giant vertical cells (VS) in the lobula plate of the blowfly Calliphora erythrocephala. J Comp Physiol A 149:163-177.

Ivanov Al, Calabrese RL (2000) Intracellular $\mathrm{Ca}^{2+}$ dynamics during spontaneous and evoked activity of leech heart interneurons: lowthreshold $\mathrm{Ca}$ currents and graded synaptic transmission. J Neurosci 20:4930-4943.

Juusola M, Uusitalo RO, Weckstrom M (1995) Transfer of graded potentials at the photoreceptor-interneuron synapse. J Gen Physiol 105:117-148.

Juusola M, Robinson HPC, de Polavieja GG (2007) Coding with spike shapes and graded potentials in cortical networks. BioEssays 29:178-187.

Juusola M, French AS (1997) The efficiency of sensory information coding by mechanoreceptor neurons. Neuron 18:959-968.

Kalb J, Egelhaaf M, Kurtz R (2006) Robust integration of motion information in the fly visual system revealed by single cell photoablation. J Neurosci 26:7898-7906.

Karmeier K, van Hateren JH, Kern R, Egelhaaf M (2006) Encoding of naturalistic optic flow by a population of blowfly motion-sensitive neurons. J Neurophysiol 96:1602-1614.

Kern R, van Hateren JH, Michaelis C, Lindemann JP, Egelhaaf M (2005) Function of a fly motion-sensitive neuron matches eye movements during free flight. PLoS Biol 3:e171.

Krapp HG, Hengstenberg B, Hengstenberg R (1998) Dendritic structure and receptive-field organization of optic flow processing interneurons in the fly. J Neurophysiol 79:1902-1917.
Kurtz R, Warzecha AK, Egelhaaf M (2001) Transfer of visual motion information via graded synapses operates linearly in the natural activity range. J Neurosci 21:6957-6966.

Laplace PS (1812) Theorie analytique des probabilitites. Paris, France: Courcier.

Nemenman I, Lewen GD, Bialek W, de Ruyter van Steveninck RR (2008) Neural coding of natural stimuli: information at sub-millisecond resolution. PLoS Comput Biol 4:e1000025.

Parsons MM, Krapp HG, Laughlin SB (2006) A motion-sensitive neurone responds to signals from the two visual systems of the blowfly, the compound eyes and ocelli. J Exp Biol 209:4464-4474.

Shu Y, Hasenstaub A, Duque A, McCormick DA (2006) Modulation of intracortical synaptic potentials by presynaptic somatic membrane potential. Nature 441:761-765.

Simmons PJ (1982) Transmission mediated with and without spikes at connections between large second-order neurones of locust ocelli. J Comp Physiol 147:401-414.

Smith SJ, Augustine GJ, Charlton MP (1985) Transmission at voltageclamped giant synapse of the squid: evidence for cooperativity of presynaptic calcium action. Proc Natl Acad Sci U S A 82:622-625.

Strausfeld NJ, Bassemir UK (1985) Lobula plate and ocellar interneurons converge onto a cluster of descending neurons leading to neck and leg motor neuropil in Calliphora erythrocephala. Cell Tissue Res 240:617-640.

Thomas JB, Wyman RJ (1984) Mutations altering synaptic connectivity between identified neurons in Drosophila. J Neurosci 4: 530-538.

Thoreson WB, Rabl K, Townes-Anderson E, Heidelberger R (2004) A highly $\mathrm{Ca}^{2+}$-sensitive pool of vesicles contributes to linearity at the rod photoreceptor ribbon synapse. Neuron 42:595-605.

Trimarchi JR, Murphey RK (1997) The shaking-B2 mutation disrupts electrical synapses in a flight circuit in adult Drosophila. J Neurosci 17:4700-4710.

Van Hateren JH, Kern R, Schwerdtfeger G, Egelhaaf M (2005) Function and coding in the blowfly $\mathrm{H} 1$ neuron during naturalistic optic flow. J Neurosci 25:4343-4352.

Warzecha AK, Kretzberg J, Egelhaaf M (1998) Temporal precision of the encoding of motion information by visual interneurons. Curr Biol 26:359-368.

Warzecha AK, Kurtz R, Egelhaaf M (2003) Synaptic transfer of dynamic motion information between identified neurons in the visual system of the blowfly. Neuroscience 119:1103-1112.

Warzecha AK, Egelhaaf M (2001) Neural encoding of visual motion in real-time. In: Vision: computational, neural, and ecological constraints (Zanker JM, Zeil J, eds), pp 239-277. Berlin: Springer Verlag.

Wilson EB (1927) Probable inference, the law of succession, and statistical inference. J Am Stat Assoc 22:209-212.

Witkovsky P, Schmitz Y, Akopian A, Krizaj D, Tranchina D (1997) Gain of rod to horizontal cell synaptic transfer: relation to glutamate release and a dihydropyridine-sensitive calcium current. J Neurosci 17:7297-7306. 